\title{
A New Method for Characterizing Biological Samples Using FTIR Microscopy with Protein Secondary Structure Estimation (IR-SSE) and ATR-TPZ Mapping
}

Ken-ichi Akao, * Hisasi Masago, * Toshiyuki Nagoshi,* Amanda L. Jenkins, ** Richard A. Larsen, ${ }^{* *}$ and Frank E. Mason**

* JASCO Corporation Spectrometry Instrument Division, 2967-5 Ishikawa-cho, Hachioji-shi Tokyo 192-8537 JAPAN

**Jasco Incorporated, 8649 Commerce Drive, Easton, MD 21601

\begin{abstract}
Many attempts have been made to obtain intact information from biological samples, such as pathological specimens, processed foods etc, without prior chemical and physical processing. FTIR microscopy is being recognized as a powerful method for identifying and characterizing different areas in heterogeneous biological samples. We have designed two different systems based on an FTIR microscopy system. (1) The IR-SSE software package is developed based on the multivariate analysis method for rapidly estimating protein secondary structure. (2) The newly developed automated accessory, ATR-TPZ is integrated to the FTIR microscope for accurately measuring reflected light from samples. This paper describes the principles of these newly developed systems and introduces some typical experimental results for biological applications.
\end{abstract}

IR-SSE-Mapping: It importance of evaluating the secondary structure of proteins without prior sample processing, such as purification or crystallization is well known. Compared to X-ray crystallography and NMR spectroscopy, the FTIR system which allows measurements to be done without sample preprocessing has significant advantages. The secondary structure of crude protein samples can be directly estimated with this system. FTIR microscopy combined with a mapping facility allows one to easily acquire a sample image along with an FTIR spectrum from heterogeneous biological samples. Since the Amide-1 peak is known to be effectively identified from the IR spectra using PCR and PLS, a dedicated analytical software package, called IR-SSE, for estimating secondary structure from the IR mapping data has been recently developed. This multivariate analysis based software allows estimation of the secondary structure of unknown samples by PCR or PLS using 17 different sets of IR standards of proteins with known secondary structure ${ }^{1)}$. Figure 1 shows actual images of the roots on a human hair and follicle. This result, $(500 \times 500 \mu \mathrm{m}$ observed area, equal to 10 x 10 points) was obtained by the JASCO IR Profile system (FTIR-470 and IRT-30 microscope). It is clearly seen from this figure that an $\alpha$-helix rich region is localized around the root. 


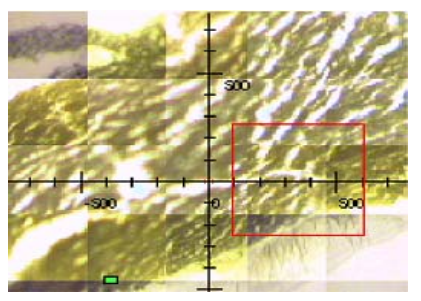

(a)

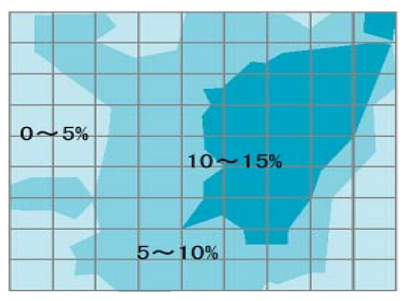

(b)

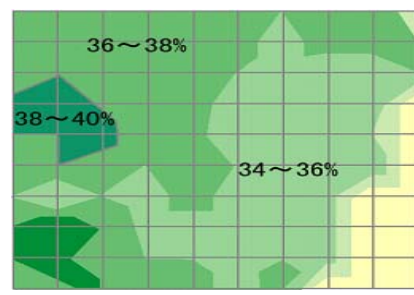

(c)

Figure 1. SSE Mapping analysis of a hair follicle (a) follicle, (b) alpha helix, and (c) beta sheet.

ATR-TPZ mapping system: Surface analysis using ATR with a microscope is an effective method for obtaining accurate IR-spectra, especially with a small sample area. The addition of the mapping system with the ATR allows the creation of an automated system for detailed surface analysis. The conventional ATR mapping method requires alternatively attaching and detaching the prism and sample, and tends to cause cross contamination especially in highly viscous samples. To solve this problem, the newly designed ATR-TPZ mapping stage controls the prism without detaching it from the sample. With this system, ATR mapping of highly viscous samples can be successfully achieved without cross contaminations. Fig.4 shows the results from a cross section of processed cheese. From these spectra and the mapping data, the distribution of the contents, water $(-\mathrm{OH}, 3264 \mathrm{~cm}-1)$ and fat $(-\mathrm{CH}, 2965 \mathrm{~cm}-1)$ is visually displayed. From this it can be determined that the difference between the spectra results from the drying conditions of the cheese.

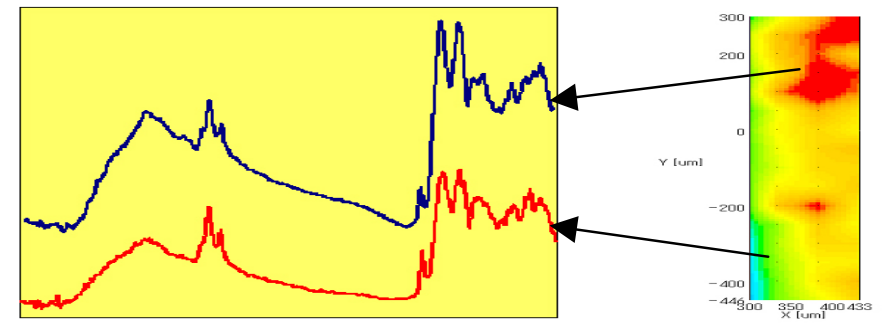

Fig.4 ATR-TPZ Mapping of cheese. Peak height ratio: 3260/2965cm-1

Summary: The IR-SSE mapping system is being applied for pathological specimen and expected to be widely used not only for clinical application but also for the food safety validation. The ATR-TPZ is being applied for food analysis and evaluating functional membranes. The combination of these two methods is expected to be utilized for many potential applications.

\section{References}

[1] Ronald W. Sarver, Analytical Biochemistry 194, 89-100 (1991)

[2] Tetsushi Yamada, et al., Clinical Cancer Research 8,2010-2014 (2002) 